\title{
In vivo Choroidal Circulation and its Watershed Zones
}

\author{
SOHAN SINGH HAYREH
}

Iowa City, Iowa, USA

\begin{abstract}
Summary
In vivo studies on choroidal vascular bed have shown that the posterior ciliary arteries (PCAs) right down to their terminal arterioles in the choroid have strictly segmental blood flow and they behave as end-arteries. These studies totally contradict the picture shown by all the postmortem cast studies of the choroidal vascular bed as a freely communicating system. The in vivo pattern helps to explain the typically localised nature of inflammatory, ischaemic, degenerative and metastatic nature of the choroidal lesions. Like other end-arterial vascular systems in the body, the choroidal vascular bed has watershed zones situated between the various PCAs, the short PCAs, the choroidal arteries, the arterioles, and the vortex veins. The end-arterial nature of the choroidal vasculature and the existence of watershed zones in the choroid are of great clinical importance; their role in the production of various ischaemic lesions in the choroid, anterior ischaemic optic neuropathy (AION) and other ischaemic disorders of the optic nerve head is discussed.
\end{abstract}

In the literature, the choroidal vascular bed is classically described as having extensive anastomoses, not only between the various choroidal arteries but also between the choroidal veins; the choriocapillaris are reported to be arranged in one plane as a continuous layer of wide lumen capillaries so that they form an uninterrupted anatomical mesh in the entire choroid. These descriptions of continuous vascular network in the choroid were based on extensive postmortem injection studies of the choroidal vascular bed, using a variety of injection materials, and recently by examining the injection casts by scanning electron microscopy. From these anatomical descriptions, it has generally been concluded that occlusion of a choroidal artery or an arteriole should not produce a choroidal infarct. However, in clinical practice, inflammatory, ischaemic, degenerative and metastatic choroidal lesions are typically localised. This puzzling discrepancy was well summarised by Duke-Elder' when he remarked that: 'The tendency for inflammatory and degenerative diseases of the choroid to show a considerable degree of selective localisation, despite the fact that anatomically the vessels would appear to form a continuous network, has given rise to speculations regarding the anatomical isolation of specific choroidal areas'.

Our in vivo experimental studies in rhesus monkeys and clinical studies in man during the past 25 years, ${ }^{2}-{ }^{30}$ using fluorescein fundus angiography as the main research technique, have radically modified our concept of the choroidal and uveal vascular bed. The in vivo studies have shown the choroidal vascular bed to be a strictly segmental and end-arterial

These studies have been supported by a research grant from the National Institutes of Health (Grant Nos. EY-1151 and EY-1576), and unrestricted grants from Research to Prevent Blindness, Inc., New York, and from the Alcon Research Institute.

Correspondence to: Sohan Singh Hayreh, MD, PhD, DSc, FRCS, Department of Ophthalmology, University Hospitals \& Clinics, Iowa City, Iowa 52242, USA. 


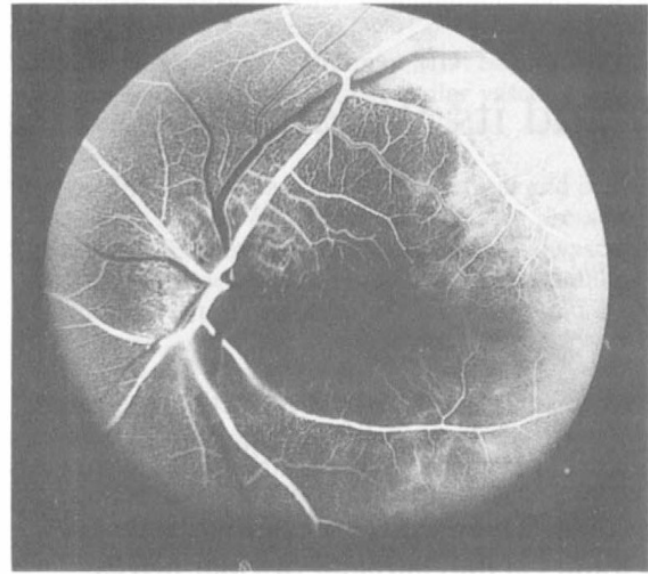

Fig. 1. Fluorescein fundus angiogram, of left eye of a 63-year-old woman with arteritic anterior ischaemic optic neuropathy (AION), shows normal filling of the area supplied by the lateral PCA but no filling of the area supplied by the medial PCA (including the entire optic disc, with no perception of light). (Reproduced from Hayreh, SS: Int Ophthalmol 1978; 1: 9-18).

system; this has helped to explain the nature of the underlying disease process in many previously obscure fundus lesions. The desription of the in vivo choroidal circulation and watershed zones given here is essentially based on our studies. ${ }^{2-30}$

\section{Anatomy of the Posterior Ciliary Arteries (PCAs):}

There is a considerable amount of misunder-

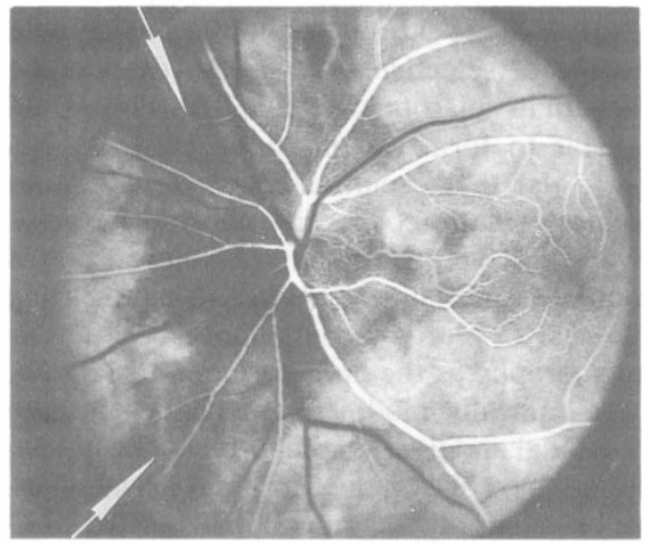

Fig. 2. Fluorescein fundus angiogram, of left eye of a 45-year-old woman with non-arteritic AION, shows non-filling of the watershed zone (indicated by arrows) between the lateral and medial PCAs. (Reproduced from Hayreh $^{26}$ ). standing among ophthalmologists on the anatomical description of the various types of PCAs. For a proper understanding of the subject, it is essential to clarify their nomenclature, origin, number and distribution. My anatomical studies ${ }^{31}$ on the PCAs in man showed that one to five posterior ciliary arteries rise from the ophthalmic artery and supply the choroid. They run forward along the optic nerve. According to the area of the choroid finally supplied by them, I have called them lateral, medial and superior PCAs, and they pierce the sclera to join the choroid lateral, medial and superior to the optic nerve respectively. The Table gives details of the number of PCAs supplying an eye. The superior PCA, when present, is usually small in size. The PCAs, during their intraorbital course and at variable distance from the eyeball, start to divide, and these branches are grouped into the following two types:

(a). Short posterior ciliary arteries pierce the sclera near the optic nerve to join the choroidal vascular bed, and supply it up to the equator. Their number depends upon the intraorbital sub-divisions of the PCAs before reaching the sclera; the short PCAs may be 10 to 20 in number.

(b). Long posterior ciliary arteries are two in number-one from the lateral PCA and the other from the medial PCA. They pierce the sclera on temporal and nasal sides respectively, anterior to the site of entry of the short PCAs. They supply a small sector of the choroid posterior to the equator on the nasal and temporal sides, in addition to the corresponding segments of the anterior uvea. ${ }^{13,29}$

The long and short PCAs have frequently been confused with the PCAs-they are in fact the branches of the PCAs and do not arise directly from the ophthalmic artery. It is essen-

Table Number of PCAs arising from the ophthalmic artery in man $^{31}$

\begin{tabular}{lrccc}
\hline Number & PCAs & $\begin{array}{c}\text { Lateral } \\
\text { PCAs }\end{array}$ & $\begin{array}{c}\text { Medial } \\
\text { PCAs }\end{array}$ & $\begin{array}{c}\text { Superior } \\
\text { PCAs }\end{array}$ \\
\hline One & $3 \%$ & $75 \%$ & $71 \%$ & $7 \%$ \\
Two & $48 \%$ & $20 \%$ & $29 \%$ & $2 \%$ \\
Three & $39 \%$ & $2 \%$ & - & - \\
Four & $8 \%$ & - & - & - \\
Five & $2 \%$ & - & - & - \\
None & - & $3 \%$ & - & $91 \%$ \\
\hline
\end{tabular}




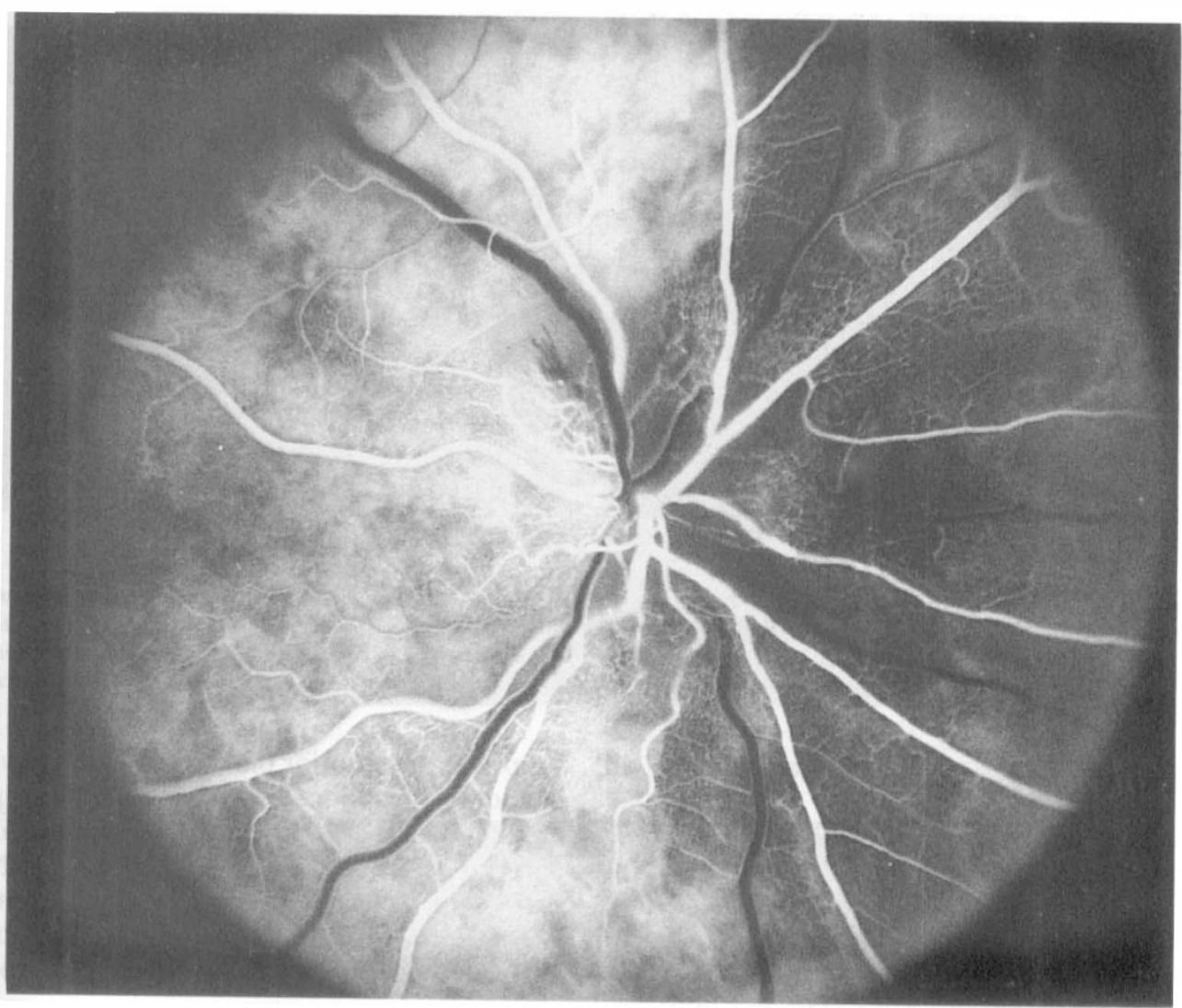

Fig. 3. Fluorescein fundus angiogram, of right eye of a 75-year-old man with AION (negative temporal artery biopsy for giant cell arteritis), shows normal filling of the area supplied by the lateral PCA (including the temporal half of optic disc) but no filling of the area supplied by the medial PCA (including the nasal half of optic disc). (Reproduced from Hayreh ${ }^{26}$ ).

tial to have a clear understanding of the various types of PCAs to avoid confusion.

\section{In vivo Choroidal Circulation:}

Fluorescein fundus angiographic studies in man and rhesus monkey, both in health and disease, have clearly shown that the choroidal blood flow is segmental in nature, as is evident from the following:

\section{A. Arterial blood flow and supply:}

1. Posterior ciliary arteries: The lateral and medial PCAs supply the corresponding parts of the choroid. . $^{3,6,14-17,19,21,23,26,30}$ There is a marked inter-individual variation in the area supplied by the two PCAs in man. ${ }^{14-16,19,23,26,30}$ The medial PCA may supply the entire nasal choroid up to the level of the fovea, including the optic nerve head (Fig. 1) or its supply may stop short nasal to the nasal peripapillary choroid (Fig. 2), so that it may take no part in the blood supply of the optic nerve head; or there may be any variation between these two extremes (Fig. 3). The lateral PCA supplies the area of the choroid not supplied by the medial PCA or vice versa (Fig. 1-3). When there are more than one medial or lateral PCAs, the area supplied by each of them may be one quadrant or only a sector (Figs. 4-6). When the superior PCA is present, it would accordingly supply a superior sector (Fig. 7). With the inter-individual variation in number (Table) and distribution by the various PCAs, we can get an extremely variable pattern of distribution in the choroid. Our clinical and experimental studies have shown that the various PCAs do not anastomose with one 


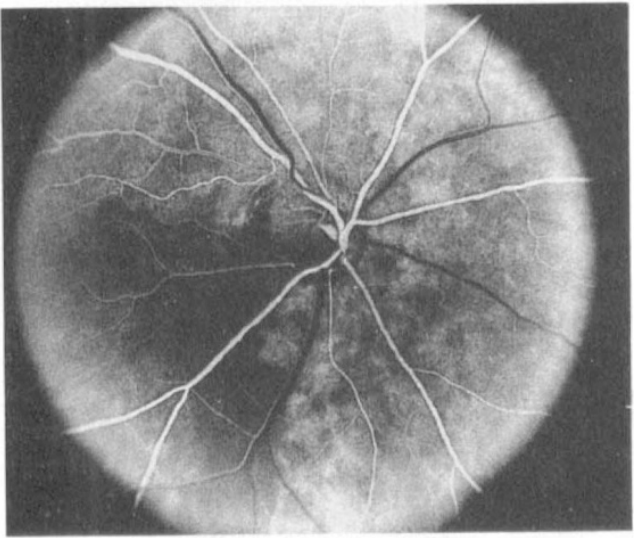

Fig. 4a.

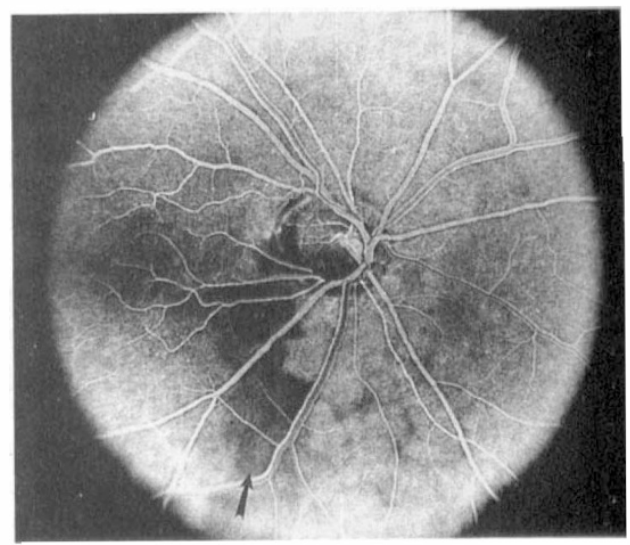

Fig. $4 b$.

Fig. 4. Fluorescein fundus angiograms, of right eye of a 69-year-old woman with low-tension glaucoma and cupping of the lower half of the optic disc with corresponding visual field defect. A-shows a filling defect in the inferior temporal quadrant of the choroid and lower half of the optic disc. B-(5 seconds after A) shows non-filling of lower half of the watershed zone (indicated by arrow) and the involved lower half of the optic disc. (Reproduced from Hayreh $^{26}$ ).

another and each behaves like an end-artery. ${ }^{3,5,6,14-17,19,21,23,26,30}$

2. Short PCAs: There are no anastomoses between the adjacent short PCAs. ${ }^{12,17}$ Each of these arteries supplies a sector of the choroid, extending usually from the posterior pole towards the equator (Figs. 8, 9). Each sector varies markedly in shape, size and location, and has irregular margins. ${ }^{12,17}$ The various sectors fit each other like pieces of a jig-saw puzzle. Further sub-divisions of the short PCAs supply correspondingly smaller segments of irregular shape and size, so that the blood supply by the various choroidal arteries has a geographic pattern-the smaller the artery, the smaller the size of the geographic piece. Finally, each terminal choroidal arteriole supplies a lobule of the choriocapillaris.

3. Long PCAs: Each artery extends radially in the horizontal meridian-one on the medial and the other on the lateral side. On the temporal side, the long PCA supplies a sector of the choroid temporal to the macular region, with it apex posteriorly (Figs. 8,9). A detailed account of the blood supply by the long PCAs is given elsewhere. ${ }^{13}$ Like the short PCAs, their smaller sub-divisions supply smaller geographic segments.

4. Peripapillary choroid: This is a very important region of the choroidal vascular bed because of its important role in the blood supply of the anterior part of the optic nerve, including the optic disc d,3,16,26,30 $^{2}$ Our experimental $^{6,12}$ and extensive clinical studies on anterior ischaemic optic neuropathy (AION) and other ischaemic disorders of the optic nerve head (Figs. 2, 4, 5, 7, 13, 14, 17, 20) have revealed that the peripapillary choroid has a segmental blood supply; this in turn is responsible for the segmental pattern of blood supply in the optic nerve head, and consequently for the well-known sectoral nature of ischaemic lesions seen in AION and other ischaemic disorders of the optic nerve head.

\section{B. Choriocapillaris:}

My fluorescein fundus angiographic studies revealed that the entire choriocapillaris bed is composed of independent small lobules. ${ }^{10,11,17,20,23}$ Each lobule is supplied by a terminal choroidal arteriole situated in the centre, and its venous drainage is by venous channels situated in the periphery of the lobule (Figs. 10, 11). My angiographic studies further revealed that each choriocapillaris lobule is an independent unit, with no anastomoses (normally) with the adjoining lobules in the living eye (Fig. 10-B), although postmortem cast preparations have all apparently revealed a continuous vascular mesh. This disparity between the in vivo and postmortem studies is important. In vivo fluores- 


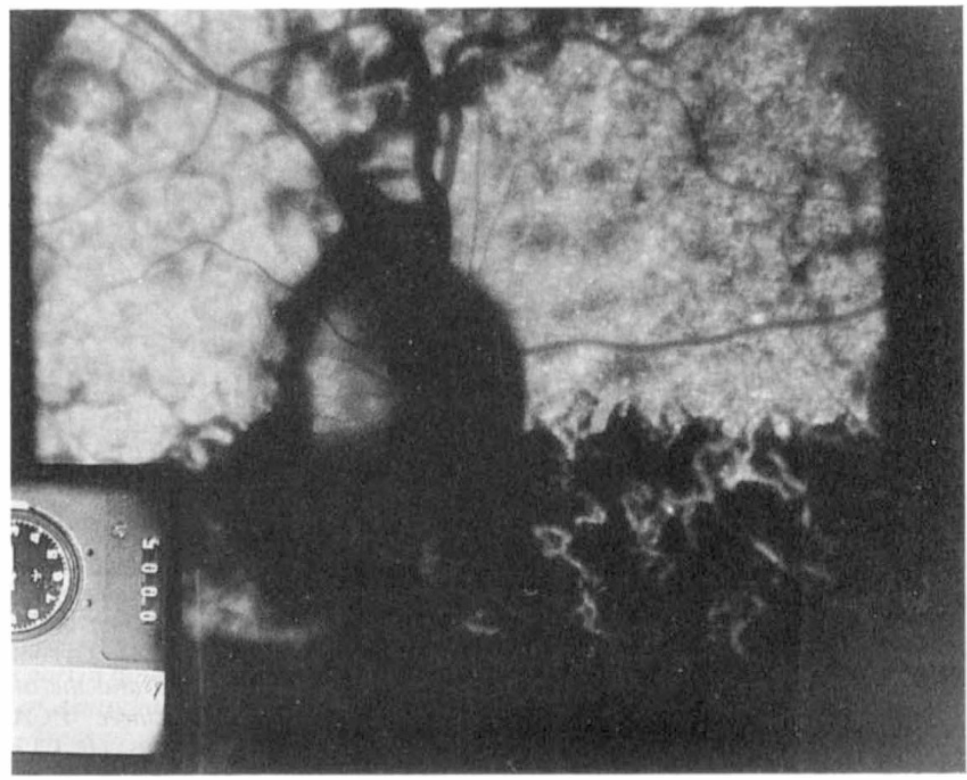

Fig. 5a.

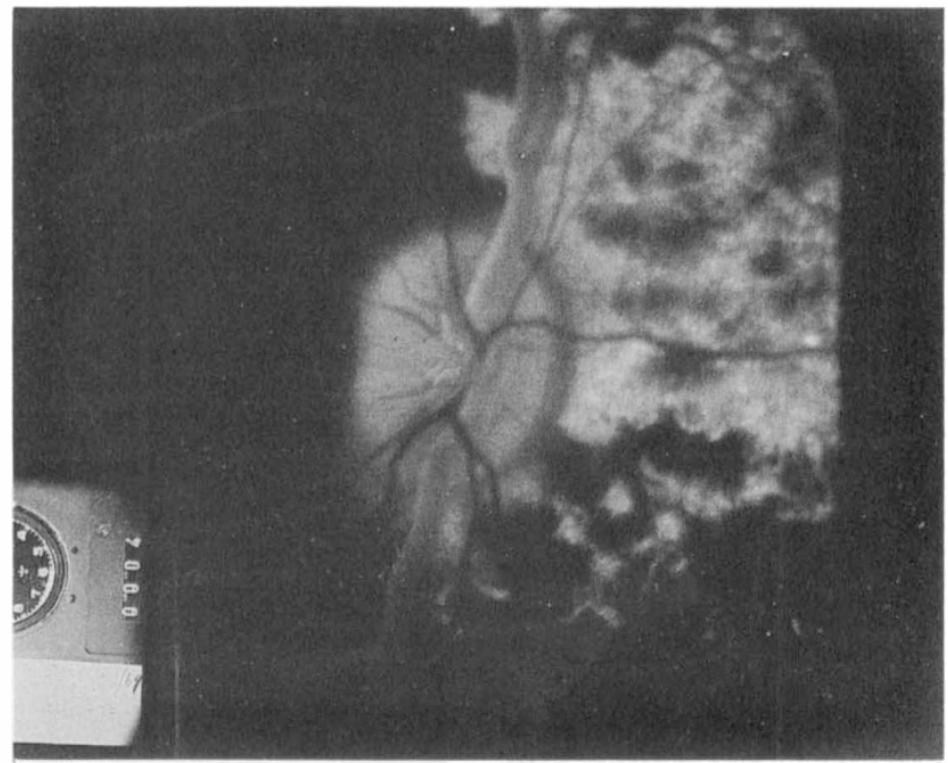

Fig. 5 b

Fig. 5. Fluorescein fundus angiograms of a normal right eye of a healthy cynomolgus monkey (after experimental occlusion of the central retinal artery to unmask vessels from the PCA-circulation in the prelaminar region of the optic disc), with four PCAs (similar to Fig. 6-E), shows: (A) After the first injection of the dye, filling of upper half of the choroid and optic disc with a well-demarcated horizontal border; (B) after the second injection of the dye, filling of superior nasal quadrant before the filling of the superior temporal quadrant of the choroid, indicating that the superior half of the choroid in $(A)$ was in fact supplied by two superior PCAs-one medial and the other lateral, and the arrow indicates the watershed zone between the two superior PCAs. The marked fluorescence of the optic disc in (B) was due to the previous injection of fluorescein. (Reproduced from Hayreh ${ }^{3}$ ). 
A
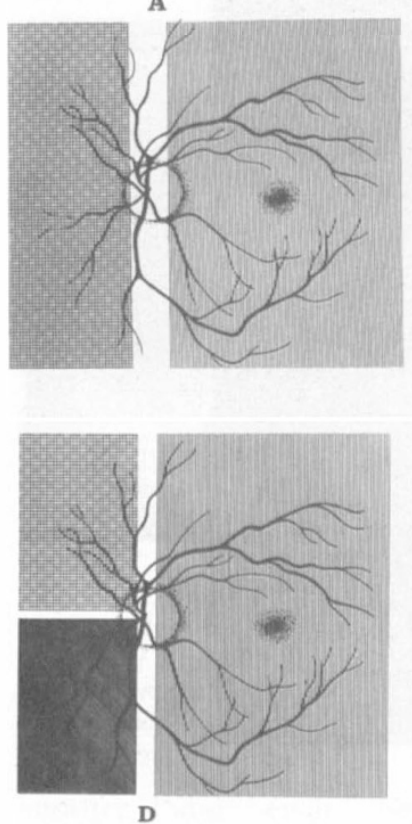
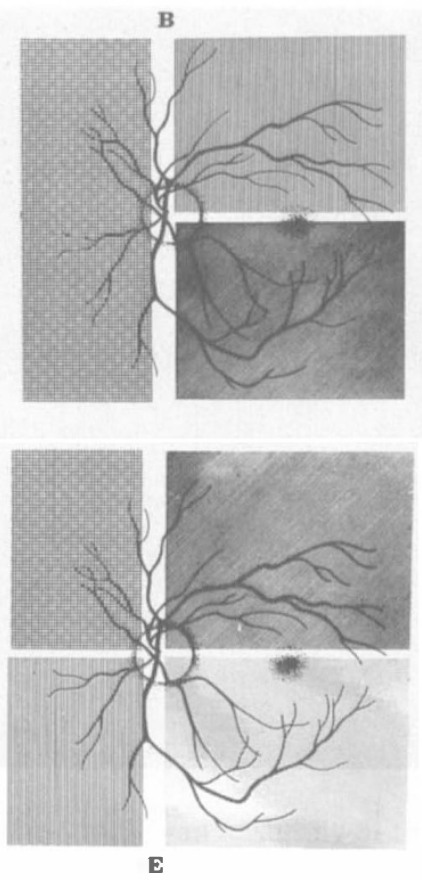

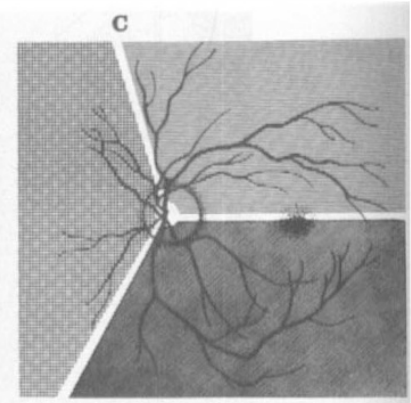

Fig. 6. Diagrammatic representations of some examples of the locations of the borders between the territories of the various PCAs: $(A)$-with two PCAs: une medial and the other lateral; $(B$ $D)$-with three PCAs in different combinations: $(B, C)$ have one medial and two lateral PCAs, and (D) one lateral and two medial; and $(E)$ with four PCAs: two medial and two lateral. (Reproduced from Hayreh ${ }^{30}$ ). cein fundus angiographic studies also revealed that the various lobules are arranged like a mosaic, with the margins of the mosaic formed by the venous channels (Figs. 10,11). The shape and size of the various choriocapillaris lobules vary in different regions of the choroid, e.g. polygonal-shaped in the posterior part and elongated in the peripheral part. The choriocapillaris are arranged more compactly at the posterior pole than in the periphery, so that they gradually become less dense towards the periphery. A detailed account of the choriocapillaris is given elsewhere. ${ }^{11,20,23}$

\section{Venous drainage:}

The venous drainage from the choroid is usually by the four vortex veins, each of which drains not only the corresponding quadrant of the choroid but also the same quadrant of the iris and ciliary body. Our in vivo studies revealed that there are normally no free anastomoses between the various vortex veins ${ }^{9,23}$ and the four vortex veins could be compared to the four major retinal veins.

This clearly shows that blood flow in the choroidal arterial, choriocapillaris and choroidal venous beds is strictly segmental, with no anastomoses between the adjacent segments at any level. ${ }^{17,20,23}$ This indicates that the PCAs and choroidal arteries, right down to the terminal choroidal arterioles, are endarteries. In this respect the choroidal vascular bed behaves exactly like the retinal vascular

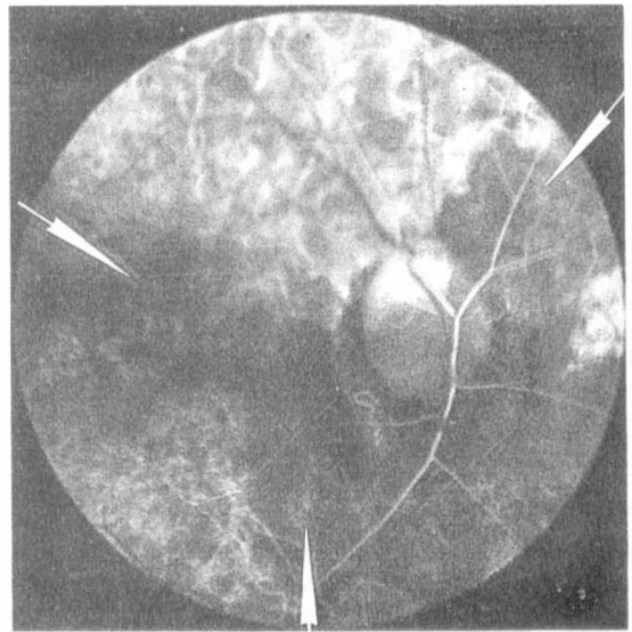

Fig. 7. Fluorescein fundus angiogram, of a normal human right eye, shows a Y-shaped watershed zone (indicated by arrows) between the superior, lateral and medial PCAs. (Reproduced from Hayreh ${ }^{14}$ ). 


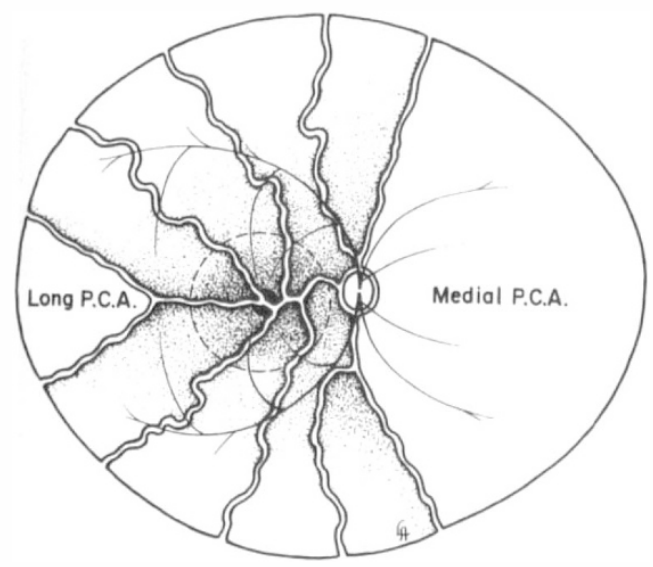

Fig. 8. Diagrammatic representation of distribution by various temporal short PCAs and their watershed zone in posterior part of fundus. Dotted circle in region of distribution of temporal short PCAs represents macular region. Areas of supply by medial PCA and temporal long PCA are also shown. (Reproduced from Hayreh $^{12}$ ).

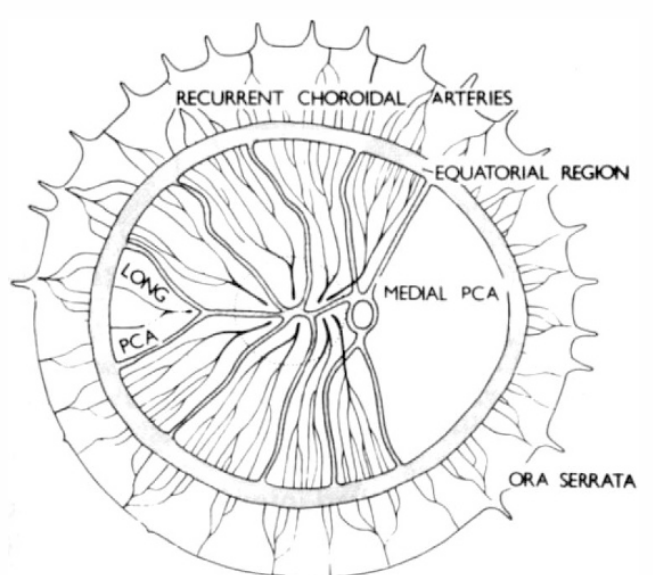

Fig. 9. Digrammatic representation of the distribution by the various ciliary arteries in the choroid and their watershed zones. The choroid posterior to the equator is supplied by the medial and lateral PCAs. In the area supplied by the lateral PCA are shown the segments supplied by the various short PCAs and the one by the long PCA, with the watershed zones between them (dotted circle in this area indicates the macular region). Recurrent choroidal arteries from the anterior ciliary arteries and supposedly the greater arterial circle of the iris supply in front of the equator. The watershed zone between the anterior and posterior choroidal arteries lies in the equatorial region. (Reproduced from Hayreh $^{20}$ ).

bed. These findings totally contradict the old concept, based on postmortem injection stud- ies, that the entire choroidal vascular bed is one continuous, freely communicating system. Clearly there is a serious disparity between the postmortem injection and the in vivo studies- the former have misled us badly all along in the past about the in vivo situation. The impressive and fascinating scanning microscopic pictures of casts published over recent years are unfortunately equally misleading in spite of their spectacular appearance. The reason for this discrepancy is that in the postmortem studies the vessels fill from all sources, irrespective of the normal blood flow pattern, when the cast material is injected under pressure, and therefore give information about the morphological conduits only, while the in vivo studies with fluorescein angiography reveal the actual pattern of the blood flow in those channels. It is also possible that in the living eye the neural supply to the potential anastomotic channels, seen on postmortem injection studies, may influence the pattern of blood flow; however, as yet, we have no definite explanation for this very important disparity. In any case, what matters clinically in explaining different vascular disorders is the in vivo circulatory pattern and not the postmortem cast findings.

\section{Watershed Zones in the Choroidal Vascular \\ Bed:}

When a tissue is supplied by two or more endarteries, the border between the territories of distribution of any two end-arteries is called a 'watershed zone'. The occurrence of such watershed zones between the various cerebral arteries is well-known, as is also the case in some other organs having end-arterial systems. The significance of the watershed zones is that in the event of a fall in the perfusion pressure in the vascular bed of one or more of the end-arteries, the watershed zone, being an area of comparatively poor vascularity, is most vulnerable to ischaemia.

From the above discussion it is evident that PCAs and their sub-divisions, right down to the choroidal arterioles, are end-arteries. The choroidal vascular bed, therefore, has watershed zones which are arranged as follows:

1. Between PCAs: Our fluorescein fundus angiographic studies in man have clearly shown the presence of watershed zones 


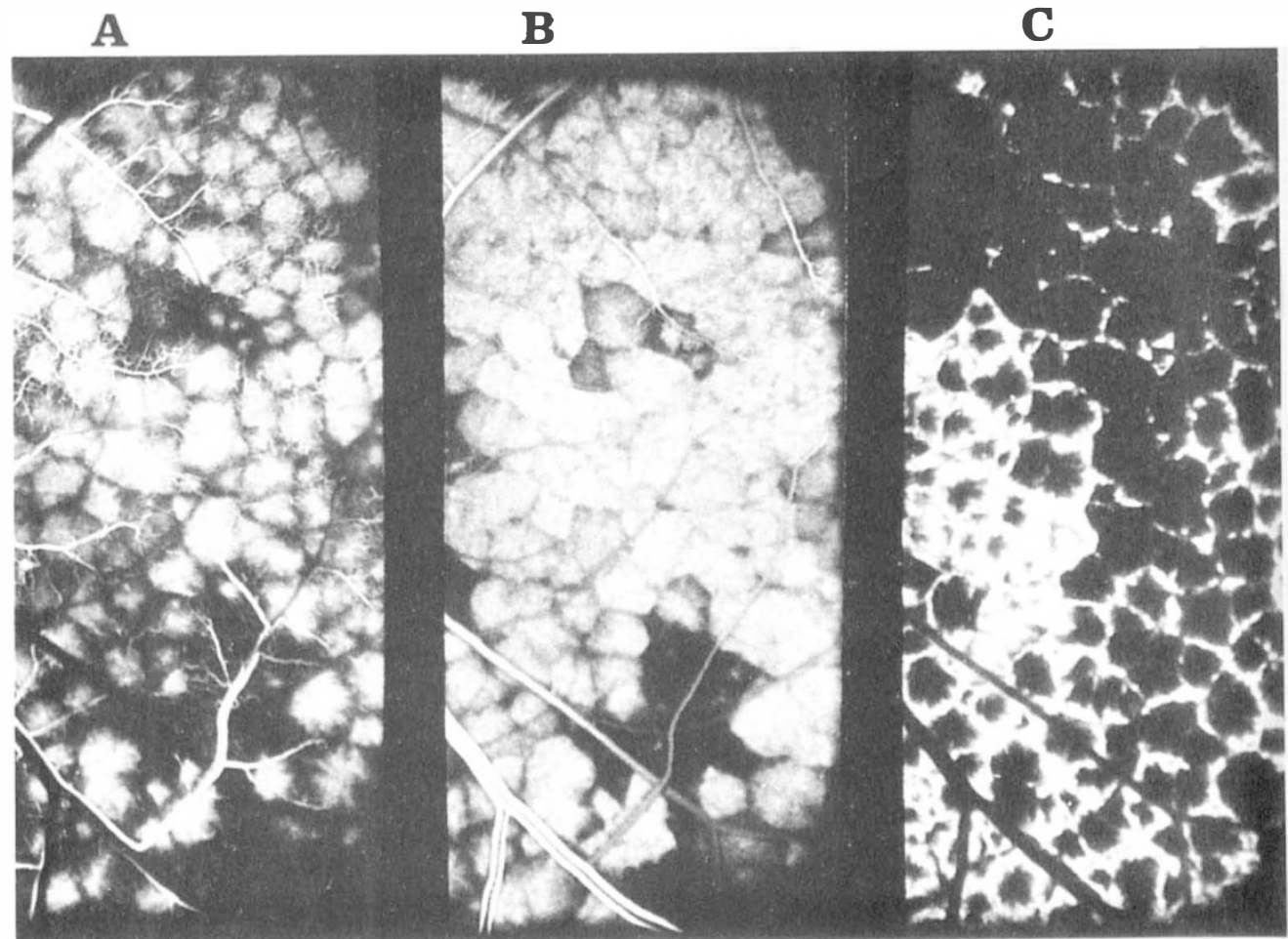

Fig. 10. Fluorescein fundus angiograms of a rhesus monkey eye at the posterior pole. A. The early arterial filling phase of the choriocapillaris, showing each lobule of the choriocapillaris (supplied by the terminal choroidal arteriole) forming a big fluorescent spot. Each spot is surrounded by a polygonal unfilled zone, producing a mosaic pattern in the choriocapillaris. B. Peak arterial filling phase. Note the extraordinarily well-defined mosaic pattern, with each unit of the mosaic an independent entity, and the isolated non-filling or slow filling of some of the lobules is clearly visible. It suggests that there is no communication between adjacent lobules. C. Venous phase of the choriocapillaris filling, showing a honeycomb pattern; the fluorescent pattern is reverse of that seen in $A$, i.e. the fluorescent areas are nonfluorescent and vice versa. (Reproduced from Hayreh ${ }^{10}$ ).

between the various PCAs. ${ }^{17,19,23,26,30}$ These can be outlined only in high quality fluorescein fundus angiography, performed at a fast speed, because normally the blood flow in the choroid is so fast that the filling pattern of the areas supplied by the various PCAs and of the watershed zones between them cannot be photographed by the slow speed of angiography equipment currently available; unless, of course, the choroidal circulation is markedly slowed down, as it often is seen in conditions including AION, carotid insufficiency and high intraocular pressure..$^{19,26,30}$ High resolution videoangiography or cineangiography can provide this information more reliably.

(i.) When there are two (medial and lateral) PCAs: As discussed above, the area of the choroid supplied by the medial and lateral
PCAs shows a marked inter-individual variation (Figs. 1-3) which must result in a wide

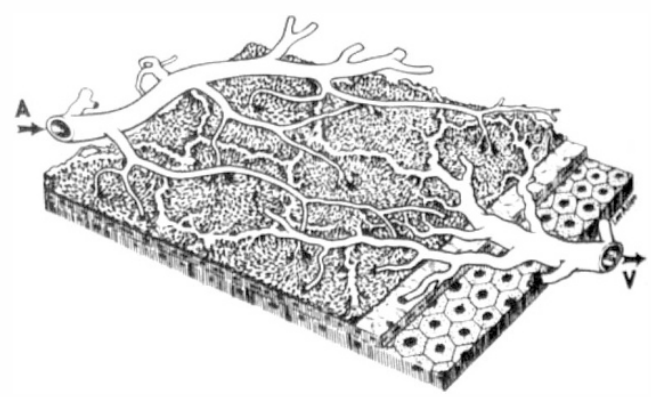

Fig. 11. A three-dimensional schematic representation of the choriocapillaris pattern. A=choroidal arteriole. V=choroidal veins. (Reproduced from Hayreh $^{11}$ ). 

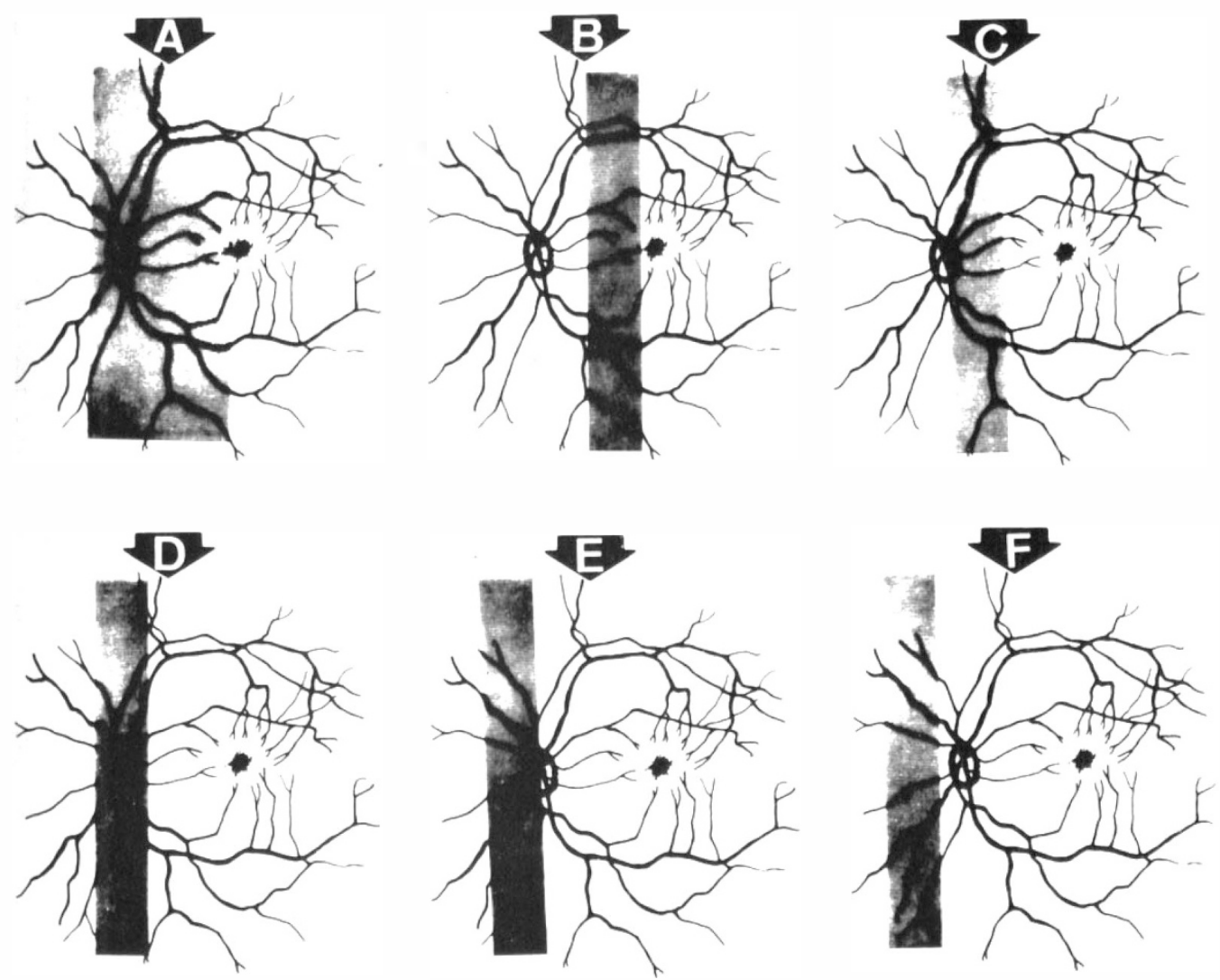

Fig. 12. Diagrammatic representation of some of the locations of the watershed zone (shaded area) between the medial and lateral PCAs in human eyes. In diagram A, the shaded area represents the overall location of the watershed zone and a watershed zone may be situated anywhere within this area. The remaining five diagrams are some examples of the variations in the location. (Reproduced from Hayreh, SS: In: Bernstein EF (ed): Amaurosis Fugax. New York: Springer-Verlag, 1988, 1-23).

variation in the location of the watershed zone between the two. Figure 12 is a diagrammatic representation of some of the locations of the watershed zone between the medial and lateral PCAs and shows that the watershed zone may:

(a) be situated temporal to the peripapillary choroid (Fig. 13),

(b) pass through the temporal peripapillary choroid (Fig. 14),

(c) pass through one or other part of the optic disc (Fig. 2, 14), or the entire optic disc may lie in the watershed zone (Fig. 15),

(d) pass through the medial peripapillary choroid (Fig. 2),

(e) various combinations of the above.

In our fluorescein fundus angiographic studies in eyes with glaucoma and low-tension-glaucoma, where we could outline the watershed zone, we found the incidence of the various locations of the watershed zone as shown in Fig. 16-the commonest $(60 \%)$ site being the temporal part of the optic disc and the adjacent peripapillary choroid.

(ii.) When there are three or more PCAs: The location of the watershed zones varies according to the number of the PCAs and their locations. With three PCAs, the watershed zone usually assumes the shape of the letter ' $Y$ ', passing through a part of the optic disc ${ }^{26,30}$ (Fig. 7); or the entire optic disc may lie in the watershed zone. ${ }^{26,30}$ Figure 6 shows diagrammatically the various combinations of watershed zones which can occur when an eye has 


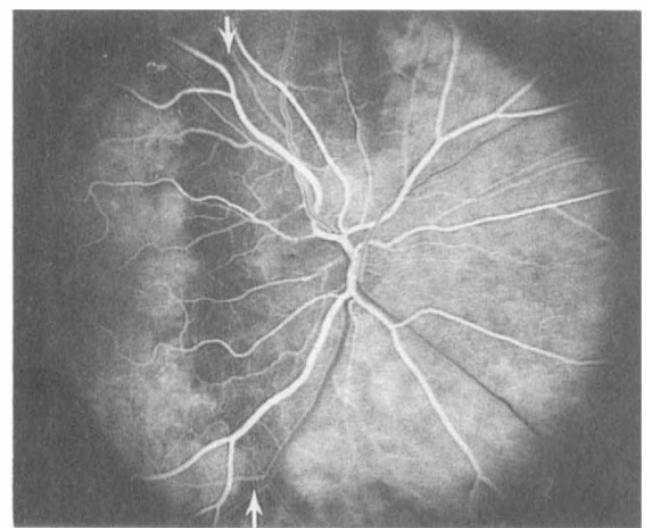

Fig. 13. Fluorescein fundus angiogram of right eye of a 78-year-old woman with giant cell arteritis and mild AION, shows non-filling of the watershed zone (indicated by arrows) between lateral and medial PCAs.

more than two PCAs; if one or more of the PCAs in such circumstances has low perfusion pressure, the eye will show a filling defect in the corresponding watershed zone, which may be vertically or obliquely oriented and involve only one-half of the watershed zone (Figs. 4, 17), in contrast to a watershed zone seen in the entire vertical length when there are only two PCAs. ${ }^{26,30}$

2. Between short PCAs: Figures 8 and 9 are a diagrammatic representation of the watershed zones between the various short PCAs, as revealed by my fluorescein angiographic

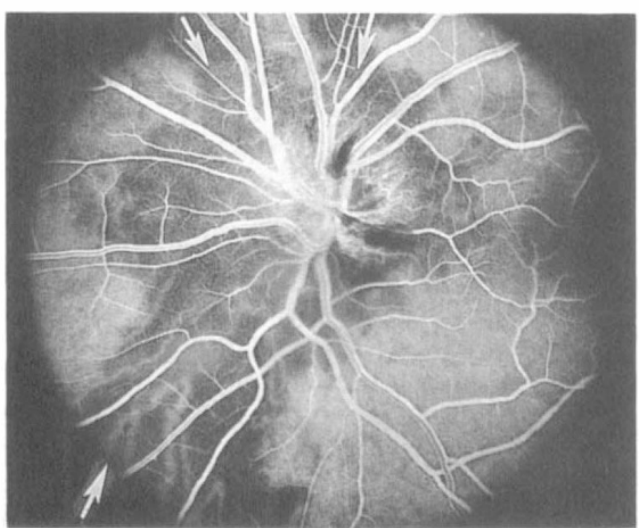

Fig. 15. Fluorescein fundus angiogram, of left eye of a 74-year-old man with arteritic AION, shows non-filling of a watershed zone (indicated by arrows) between the lateral and medial PCAs. (Reproduced from Hayreh $^{26}$ ).

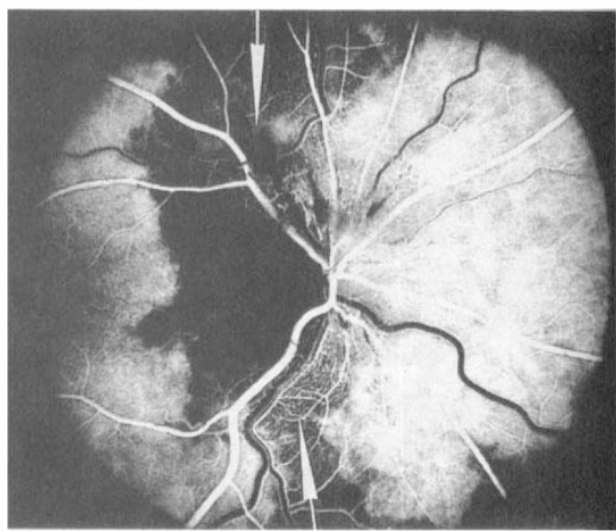

Fig. 14. Fluorescein fundus angiogram, of right eye of a 60-year-old man withnon-arteritic AION, shows nonfilling of the watershed zone (indicated by arrows) between the lateral and medial PCAs. (Reproduced from Hayreh ${ }^{26}$ ).

studies in experimental occlusion of the temporal short PCAs. ${ }^{12}$ These studies revealed that the area supplied by each short PCA has a well-defined margin, with watershed zones
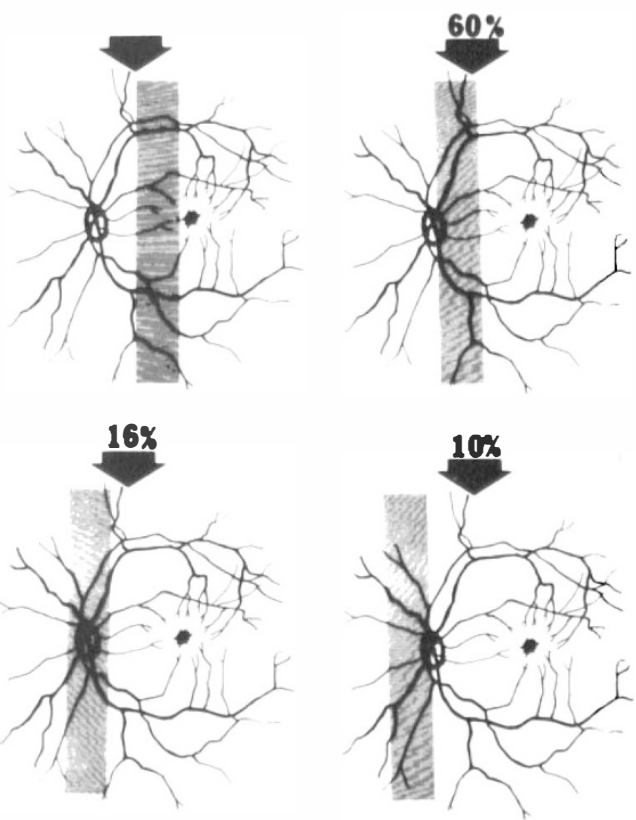

Fig. 16. Diagrammatic representation of some of the locations of the watershed zone (shaded area) between medial and lateral PCAs and their incidence in human eyes with primary open angle glaucoma and lowtension glaucoma. (Reproduced from Hayreh ${ }^{30}$ ). 


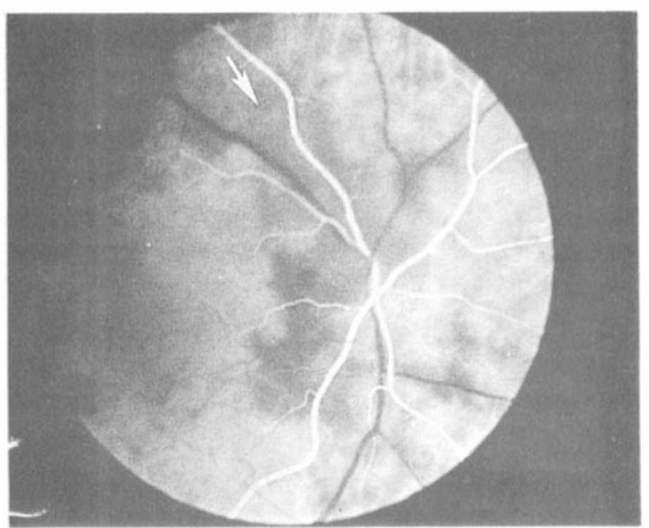

Fig. 17. Fluorescein funds angiogram, of right eye of a 72-year-old man with arteritic AION, shows a nonfilling of the upper half of the watershed zone (indicated by arrow) and involved upper half of the optic disc. (Reproduced from Hayreh ${ }^{15}$ ).

situated between adjacent short PCAs. All the temporal short PCAs enter the eyeball in the macular region and spread out to the periphery of the fundus to supply the temporal half of the choroid ${ }^{12.20 .23 .25}$ (Fig. 18). It is, therefore, natural that most of the segments of the choroid supplied by the temporal short PCAs and their watershed zones meet in the macular region. This was a consistent pattern in my studies. It is well-established that an area where numerous watershed zones meet is usually an area of comparatively poor vascularity and in the event of circulatory disorders, most vulnerable to ischaemia.

3. Between the short and long PCAs: Figures 8 and 9 show the area of supply by the temporal long PCA. My experimental studies revealed that there are no anastomoses between the long PCA and the adjacent short PCAs. ${ }^{13}$ Thus there is a watershed zone present between the long PCA and its adjacent short PCAs.

4. Between the PCAs and the anterior ciliary arteries: Our experimental and clinical studies dealing with PCA occlusion have clearly shown that there are no anastomoses between the posterior and anterior ciliary arteries, because in the event of PCA occlusion the choroid does not fill by extension from the anterior ciliary arteries. ${ }^{6.1+-17.19-21.23 .26 .311}$ Similarly, our clinical and experimental studies on anterior ciliary artery occlusion (fol-

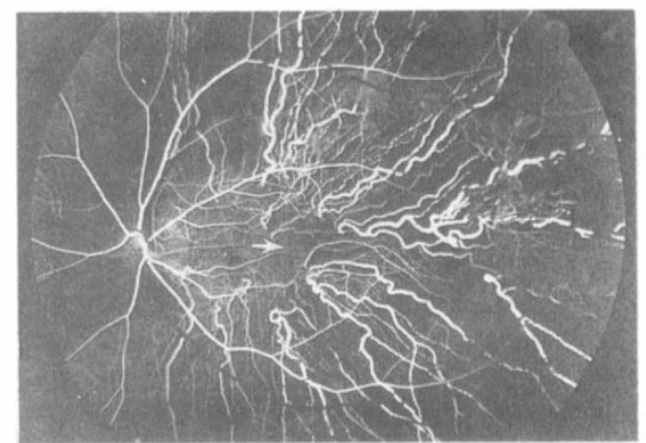

Fig. 18. Fluorescein fundus angiogram of a normal human eye, showing the sites of entry of the short PCAs and their course in the choroid. Arrow marks the centre of the macular region. Note that no artery lies in the centre of the macular region. (Reproduced by the kind courtesy of Dr P. Amalric: Int. Ophthalmol. 1983; 6: 149-53).

lowing resection of the various recti) revealed that PCAs do not safeguard the anterior uvea from development of anterior segment ischaemia in these eyes. ${ }^{18.29}$ The watershed zone between the anterior and posterior ciliary arteries is situated in the equatorial region of the choroid ${ }^{20.23}$ (Fig. 9).

5. Between the choriocapillaris lobules: The watershed zones between the various lobules of the choriocapillaris are arranged like a honeycomb ${ }^{11.17 .20 .23}$ (Fig. 10).

6. Between the vortex veins: Our experimental studies on vortex vein occlusion in rhesus

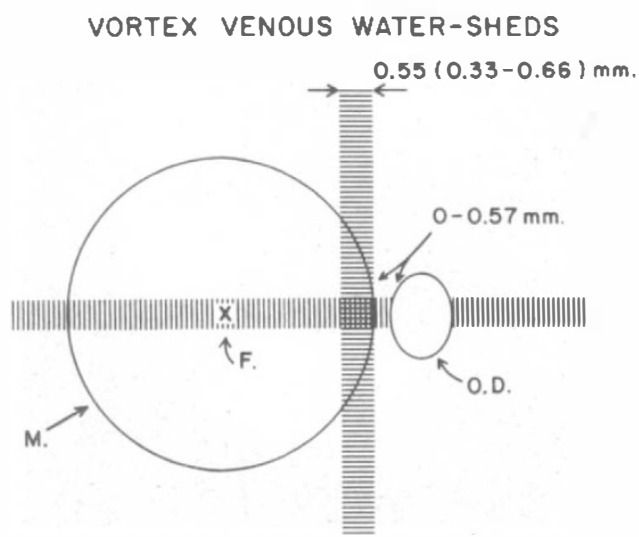

Fig. 19. Diagrammatic representation of watershed zones between the various vortex veins in rhesus monkeys. $F \&$ \& fovea. $M=$ macular region. $O D=$ optic disc. (Reproduced from Hayreh ${ }^{12}$ ). 
A

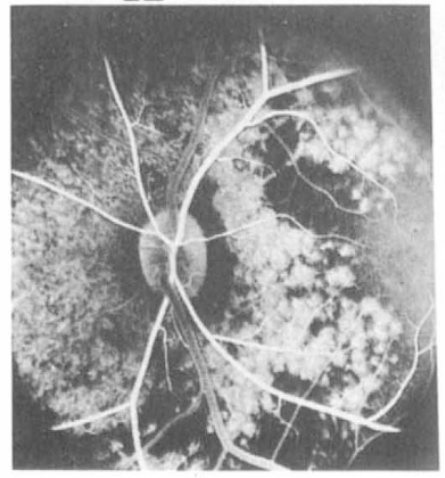

B

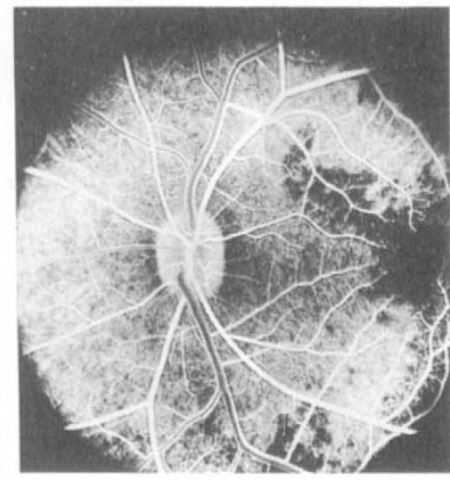

C

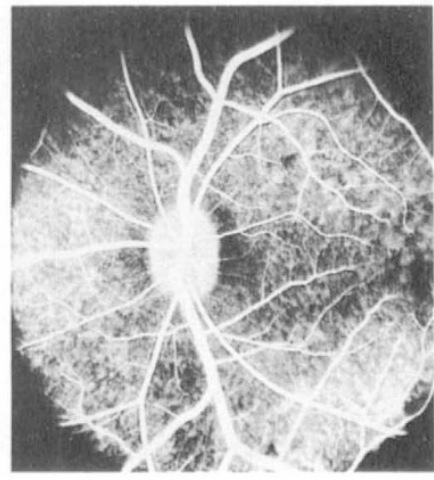

Fig. 20. Fluorescein fundus angiograms in left eye of a rhesus monkey, after reduced perfusion pressure in the eye, 9.1 $(A), 11.2(B)$ and 17.2(C) seconds after intravenous injection of fluorescein. Note slow filling of the choroid, particularly marked in the watershed zones between the various temporal short PCAs and the macular region in $A$ and $B$. (Reproduced from Hayreh ${ }^{20}$ ).

monkeys revealed that the various vortex veins show no free anastomoses and that the watershed zones between the four vortex veins extend antero-posteriorly through the entire length of the uveal tract-a horizontal watershed zone between the upper and lower vortex veins passes through the optic disc and macular region, while a vertical watershed zone between the temporal and nasal vortex veins passes between the optic disc and macular region ${ }^{9,17,23}$ (Fig. 19).

\section{Clinical Importance of the in vivo Choroidal Vascular Pattern and of the Watershed Zones in it:}

The PCAs supply the following tissues:

(i). Choroid: This is supplied up to the equator.

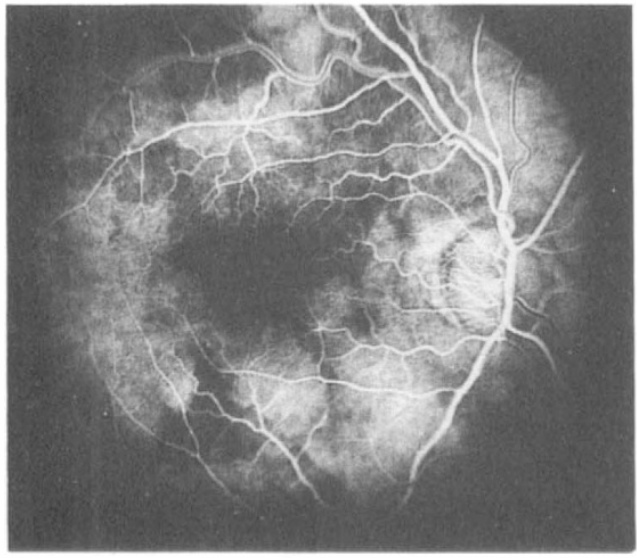

Fig. 21 a.

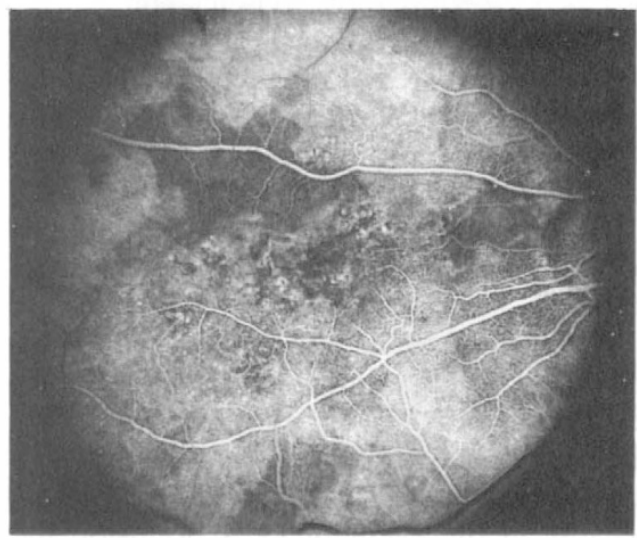

Fig. $21 b$.

Fig. 21. Fluorescein fundus angiograms of the posterior pole of two patients with early senile macular degeneration, showing delayed filling of the watershed zones between the temporal short PCAs and in the center of the macular region. (Reproduced from Hayreh ${ }^{25}$ ). 


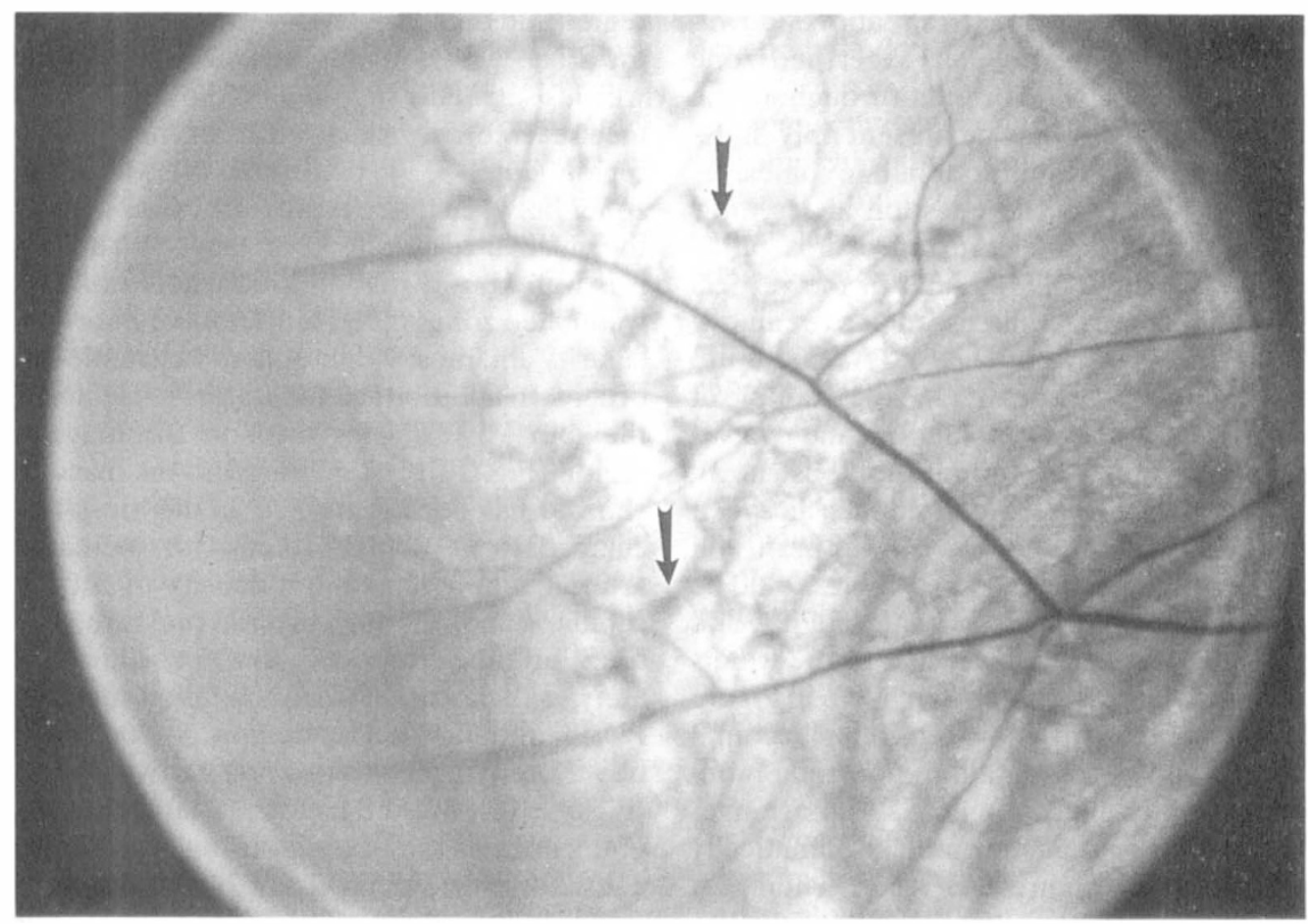

Fig. 22. Reticular pigmentary degeneration (arrows) in the equatorial region of a patient with senile macular degeneration. The pigment accumulates in lines forming irregular polygons. (Reproduced from Hayreh ${ }^{20}$ ).

(ii). Anterior part of the optic nerve: PCAs are the main source of blood supply to the optic nerve head and the adjacent retrolaminar region, mostly via the peripapillary choroid and to a small extent directly through branches from the short PCAs.

(iii). Retina: The choroid supplies the overlying retina to a depth of $130 \mathrm{u}$, including the retinal pigment epithelium and adjacent outer retina up to the outer part of the inner nuclear layer. When a cilio-retinal artery is present, the entire thickness of the retina is supplied in the distribution of the cilio-retinal artery.

A. Important role played by the location of PCAs' watershed zones in relation to the optic disc in the production of AION and other ischaemic disorders of the optic nerve head: This is an extremely important subject in any discussion of ischaemic disorders of the optic nerve head, because PCA-circulation is the main source of blood supply to the anterior part of the optic nerve, as was revealed by our clinical studies on AION, glaucoma and lowtension glaucoma. ${ }^{26,30}$ Our studies indicate that the location of the watershed zone deter- mines the vulnerability of the corresponding part of the optic disc to ischaemia (Fig. 12). If the watershed zone is located away from the optic disc, the optic nerve head is comparatively less vulnerable to ischaemia than if the watershed zone passes through it. The part of the optic disc that lies in the watershed zone is more vulnerable to ischaemia than the part that does not. When the entire optic disc lies in the centre of the watershed zone, that disc is most vulnerable to ischaemia (Fig. 15). We have seen this in eyes with AION where the part of the optic disc involved was the part with the watershed zone in it. The location of the watershed zone in relation to the optic disc would in turn depend upon the distribution pattern of the various PCAs. The marked variation in the area supplied by the various PCAs described above would consequently influence the location of the watershed zone.

I am frequently asked, how can one explain the frequent occurrence of an altitudinal defect in many eyes with AION, when in most of the angiograms shown for the watershed zone, it is usually a complete vertical defect 
extending all the way from above downwards? The answer is that watershed zone defects are not always present throughout the vertical length but may be present only in the superior (Fig. 17) or inferior half in some eyes (Fig. 4). Naturally the question arises, how can one explain the occurrence of a filling defect involving only one-half of the vertical watershed zone? As discussed above, an eye can have one or two medial PCAs and up to three lateral PCAs. Let us take the example of an eye which has one medial PCA and two lateral PCAs (superior and inferior), as shown diagrammatically in Fig. 6-B; in such an eye, if the inferior lateral PCA has a lower perfusion pressure than the other PCAs, there would be delayed filling of the inferior lateral PCA and a filling defect in the lower half of the watershed only-such a situation is shown in Figure 4 from an eye with low-tension glaucoma. Figure 5 shows angiogram of a normal monkey eye which had four PCAs-an arrangement similar to that shown diagrammatically in Figure 6-E: Figure 5-B records filling of only the superior medial PCA, and Figure 5-A of both superior medial and superior lateral PCAs, with the watershed zone located between the two superiorly (indicated by an arrow), and no filling of the inferior medial and inferior lateral PCAs. Figure 6 shows diagrammatically the various combinations which can occur when an eye has more than two PCAs, to account for the development of filling defect in only one-half of the watershed zone, which may be vertically or obliquely oriented. The corresponding part of the optic disc would be involved by ischaemia. We have actually seen most of these examples in our clinical studies; the others remain as yet speculative.

The end-arterial nature of the PCA-circulation also explains the development of only a sectoral ischaemia in the optic nerve head, which is typically seen in AION and other ischaemic disorders of the optic nerve head.

B. Importance of location of multiple watershed zones of the short PCAs in the macular choroid and its possible role in production of various macular ischaemic lesions and agerelated macular degeneration: As discussed above, all the temporal short PCAs pierce the sclera and join the choroid in the macular region and then run radially towards the equator (Figs. 9, 18), so that the apical parts of the various segments supplied by the short PCAs meet each other in the centre of the macular region (Figs. 8, 9); therefore, the watershed zones between the several short PCAs meet in the macular region. ${ }^{12,17,20,23,25}$ As stated above, it is well known that an area where multiple watershed zones meet, is an area of poor vascularity and most vulnerable to ischaemic disorders. In addition, all the watershed zones of the four vortex veins meet in the macular region (Fig. 19). In the literature the macular choroid has been described as the most vascular part of the choroid, with increased arterial supply, maximum density of choriocapillaris and very high arterial pressure. The basis of this erroneous concept, unfortunately, is the location of all the temporal short PCAs initially in this region, before they travel centrifugally to their respective sectors (Figs. 9, 18); but the fact that a large number of big arteries are passing through this region does not mean their entire blood supply is meant for the macular choroid.

There is other evidence suggesting that the macular choroid is more vulnerable to ischaemic disorders than other parts of the choroid-for instance:

(a). In our experimental studies on malignant arterial hypertension in rhesus monkeys, choroidal ischaemia was the main feature of the hypertensive choroidopathy, seen almost invariably in these animals. ${ }^{28}$ The choroidal ischaemic lesions were most prominent in the macular region and fluorescein fundus angiography revealed a marked, selective delayed filling of the macular choroid, particularly of its central part.

(b). In our experimental studies in rhesus monkeys, where we reduced the perfusion pressure in the ocular vessels by lowering the systemic blood pressure, fluorescein angiography revealed delayed filling of the watershed zones in the macular choroid, and of the central choriocapillaris ${ }^{20,25}$ (Fig. 20).

(c). There have been reports of selective localised senile atrophy of the choriocapillaris and in some cases even of large choroidal vessels, in the macular choroid. ${ }^{32,34}$

In the light of all this information, it would be logical to consider that in eyes with marked 
generalised atherosclerosis and arteriosclerosis of the choroidal vessels, the macular choroid would be most vulnerable to ischaemic disorders, by virtue of the numerous watershed zones meeting there. ${ }^{12,20,25}$ In fluorescein angiography of eyes with age-related macular degeneration, we have seen in some eyes prolonged delay in the filling of the watershed zones in the macular region ${ }^{20,25}$ (Fig. 21). Recently, Chen et al. ${ }^{35}$ have shown delayed choriocapillaris filling on fluorescein fundus angiography in age-related macular degeneration with decreased visual acuity. They 'concluded that chronic choroidal vascular compromise is an important cause of visual impairment in age-related macular degeneration.' Thus, all the evidence collectively seems to support my original hypothesis, postulated about 15 years ago, ${ }^{12}$ that the macular choroid is more vulnerable to chronic ischaemia than any other part of the posterior choroid and that chronic choroidal ischaemia may play an important role in the production of age-related macular degeneration and associated neovascularisation. Similarly, in eyes with generalised chronic choroidal ischaemia, a reticular pigmentary degeneration is frequently seen in the equatorial region (in the region of the equatorial watershed zone between the anterior and posterior ciliary circulation), particularly on the nasal side (Fig. 22). Eyes with senile macular generation frequently show this equatorial reticular degeneration, thereby further suggesting chronic ischaemia as an important factor in the production of age-related macular degeneration.

C. Role of end-arterial nature of choroidal vasculature in production of acute ischaemic lesions in the choroid and optic nerve head: These lesions have been seen in a number of systemic diseases, including malignant arterial hypertension, collagen vascular diseases, haematologic diseases, disseminated intravascular coagulopathy, thrombotic thrombocytopenic purpura, giant cell arteritis, carotid artery disease, cardiac valvular lesions and a host of other conditions. ${ }^{24} \mathrm{We}$ have also produced these experimentally by PCA occlusion ${ }^{5,7,8,21,24}$ and by malignant arterial hypertension, ${ }^{27.28}$ in rhesus monkeys. In the optic nerve head they produce AION.
The acute choroidal ischaemic lesions, depending upon the size of the choroidal artery or arteriole involved, severity of ischaemia and stage of evolution, have polymorphic clinical presentation but they represent a continuous spectrum of disease which can be divided into the following separate clinical entities for descriptive purposes. ${ }^{24}$

(a). Elschnig's spot: This localised choroidal infarct is produced by occlusion of a terminal choroidal arteriole.

(b). Multifocal acute ischaemic choroidopathy: This entity was originally thought to represent a disorder of the retinal pigment epithelium and consequently was called 'acute posterior multifocal placoid pigment epitheliopathy'. ${ }^{36}$ The available evidence indicates that it is in fact an acute ischaemic lesion due to focal occlusion of small choroidal arterioles. ${ }^{24}$ In view of this, it would be more appropriate to call it 'multifocal acute ischaemic choroidopathy'.

(c). Geographic choroidopathy: The ophthalmoscopic, fluorescein fundus angiographic and evolution patterns of those lesions are typically those seen in focal acute choroidal ischaemia, ${ }^{24}$ and as such this would seem to represent an acute occlusive disorder of larger choroidal arterioles or small arteries.

(d). Triangular sector-shaped lesions: Amalric $^{37}$ described triangular wedge-shaped areas of pigmentary disturbance in the fundus, with their bases loward the equator and apices toward the posterior role. I have seen these lesions in our experimental ${ }^{7,21}$ and clinical ${ }^{16,24}$ studies. These are produced by occlusion of major choroidal arteries.

(e). Extensive regional choroidal infarcts: These are produced by PCA occlusion; we have produced them experimentally $y^{7.21}$ and seen them clinically. ${ }^{16.24}$

(f). Conditions associated with serous retinal detachment: This is produced by ischaemic dysfunction of the retinal pigment epithelium, with or without demonstrable frank epithelial infarction, so that the retinal pigment epithelium loses its blood-retinal barrier property and allows subretinal fluid accumulation. ${ }^{24,28}$

(g). Post-operative acute choroidal ischaemia: This is seen in some eyes following vitrectomy and other closed-system ocular procedures 
where the intraocular pressure is kept very high during prolonged surgery. ${ }^{22,24}$

All these conditons are discussed at length elsewhere. $^{24}$

D. Acute occlusive disorders of the vortex veins: We have very little clinical information on this subject. Our experimental occlusion of the vortex veins ${ }^{9}$ indicates that it may play an important role in the production of some of the major complications following encircling procedure and cyropexy for retinal detachment, including raised intraocular pressure, anterior segment ischaemia, choroidal and intraocular haemorrhages and the so-called 'anterior uveitis'. The subject is discussed at length elsewhere. ${ }^{9,24}$

I am grateful to my wife Shelagh for her help with the manuscript, to Mrs Ellen Ballas and Mrs Georgiane Parkes-Perret for their secretarial help, and to the photography department for the illustration.

\section{References}

${ }^{1}$ Duke-Elder S: System of Ophthalmology. Vol. 2. London. Kimpton, 1961: 351.

${ }^{2}$ Hayreh SS: Blood supply of the optic nerve and its role in optic atrophy, glaucoma, and oedema of the optic disc. Br J Ophthalmol 1969, 53: 721-48.

${ }^{3}$ Hayreh SS: Pathogenesis of visual field defects. Role of the ciliary circulation. Br J Ophthalmol 1970, 54: $289-311$.

${ }^{4}$ Hayreh SS, Revie IHS, Edwards J: Vasogenic origin of visual field defects and optic nerve changes in glaucoma. Br J Ophthalmol 1970, 54: 461-72.

${ }^{5}$ Hayreh SS: Posterior ciliary arterial occlusive disorders. Trans Ophthalmol Soc UK 1971, 91: 291-303.

${ }^{6}$ Hayreh SS and Baines JAB: Occlusion of the posterior ciliary artery. I. Effects on choroidal circulation. Br J Ophthalmol 1972, 56: 719-35.

${ }^{7}$ Hayreh SS and Baines JAB: Occlusion of the posterior ciliary artery. II. Chorioretinal lesions. $\mathrm{Br} \mathrm{J}$ Ophthalmol 1972, 56: 736-53.

${ }^{8}$ Hayreh SS and Baines JAB: Occlusion of the posterior ciliary artery. III. Effects on the optic nerve head. Br J Ophthalmol 1972, 56: 754-64.

${ }^{9}$ Hayreh SS and Baines JAB: Occlusion of the vortex veins. An experimental study. Br J Ophthalmol 1973, 57: 217-38.

${ }^{10}$ Hayreh SS: Recent advances in fluorescein fundus angiography. Br J Ophthalmol 1974, 58: 391-412.

${ }^{11}$ Hayreh SS: The choriocapillaris. Graefes Arch Clin Exp Ophthalmol 1974, 192: 165-79.

${ }^{12}$ Hayreh SS: Submacular choroidal vascular pattern. Experimental fluorescein fundus angiographic studies. Graefes Arch Clin Exp Ophthalmol 1974, 192: $181-96$.

${ }^{13}$ Hayreh SS: The long posterior ciliary arteries. An experimental study. Graefes Arch Clin Exp Ophthalmol 1974, 192: 197-213.

${ }^{14}$ Hayreh SS: Anterior ischaemic optic neuropathy. I. Terminology and pathogenesis. Br J Ophthalmol 1974, 58: 955-63.

${ }^{15}$ Hayreh SS: Anterior ischaemic optic neuropathy. II. Fundus on ophthalmoscopy and fluorescein angiography. Br J Ophthalmol 1974, 58: 964-80.

${ }^{16}$ Hayreh SS: Anterior ischemic optic neuropathy. New York. Springer-Verlag, 1975.

${ }^{17}$ Hayreh SS: Segmental nature of the choroidal vasculature. Br J Ophthalmol 1975, 59: 631-48.

${ }^{18}$ Hayreh SS and Scott WE: Fluorescein iris angiography. II. Disturbances in iris circulation following strabismus operation on the various recti. Arch Ophthalmol 1978, 96: 1390-1400.

${ }^{19}$ Hayreh SS: Anterior ischemic optic neuropathy. Arch Neurol 1981, 38: 675-78.

${ }^{20}$ Hayreh SS: Controversies on submacular choroidal circulation. Ophthalmologica 1981, 183: 11-19.

${ }^{21}$ Hayreh SS and Chopdar A: Occlusion of the posterior ciliary artery. V. Protective influence of simultaneous vortex vein occlusion. Arch Ophthalmol 1982, 100: 1481-91.

${ }^{22}$ Hayreh SS: Choroidal ischemia. Retina 1982, 2 : 191-2.

${ }^{23}$ Hayreh SS: Physiological anatomy of the choroidal vascular bed. Int Ophthalmol 1983, 6: 85-93.

${ }^{24}$ Hayreh SS: Acute occlusive disorders of the choroidal vasculature. Int Ophthalmol 1983, 6: $139-48$.

${ }^{25}$ Hayreh SS: Macular lesions secondary to choroidal vascular disorders. Int Ophthalmol 1983, 6: 161-70.

${ }^{26}$ Hayreh SS: Inter-individual variation in blood supply of the optic nerve. Its importance in various ischemic disorders of the optic nerve head, and glaucoma, low-tension glaucoma and allied disorders. Doc Ophthalmol 1985, 59: 217-46.

${ }^{27}$ Hayreh SS, Servais GE, Virdi PS: Fundus lesions in malignant hypertension. V. Hypertensive optic neuropathy. Ophthalmology 1986, 93: 74-87.

${ }^{28}$ Hayreh SS, Servais GE, Virdi PS: Fundus lesions in malignant hypertension. VI. Hypertensive choroidopathy. Ophthalmology 1986, 93: 1383-1400.

${ }^{29}$ Virdi PS and Hayreh SS: Anterior segment ischemia after recession of various recti. An experimental study. Ophthalmology 1987, 94: 1258-71.

${ }^{30}$ Hayreh SS: Blood supply of the optic nerve head in health and disease. In Lambrou GN, Greve EL eds. Ocular blood flow measurements in glaucoma. Amsterdam: Kugler \& Ghedini Publications 1989: 1-46.

${ }^{31}$ Hayreh SS: The ophthalmic artery. III. Branches. $B r$ J Ophthalmol 1962, 46: 212-47.

${ }^{32}$ Kornzweig AL: Changes in the choriocapillaris associated with senile macular degeneration. Ann Ophthalmol 1977, 9: 753-64.

${ }^{33}$ Sarks SH: Ageing and degeneration in the macular region: A clinicopathological study. $\mathrm{Br} J O$ phthal. mol 1976, 60: 324-41.

${ }^{34}$ Sarks SH: Changes in the region of the choriocapilla- 
ris in ageing and degeneration. Acta XXIII Conc. Ophthalmol. Kyoto, 1978. Excerpta Medica, Amsterdam 1979: 228-38.

Chen JC, Fitzke FW, Pauliekhoff D, Bird AC: Poor choroidal perfusion is a cause of visual morbidity in age-related macular degeneration. Invest Ophthalmol Vis Sci 1989, 30 (Suppl): 153.
${ }^{36}$ Gass JDM: Acute posterior multifocal placoid pigment epitheliopathy. Arch Ophthalmol 1968, 80: 177-85.

${ }^{37}$ Amalric P: Circulation choroidienne. In Amalric P ed. Proceedings of the International Symposium on Fluorescein Angiography, Albi 1969, Basel: Karger 1971: 193-203. 